\title{
Multisystem inflammatory syndrome in children related to COVID-19: An update regarding the presentation of two critically ill patients
}

Pedro Taffarel, M.D. ${ }^{a}$, Facundo Jorro Barón, M.D. ${ }^{a}$, Ana Paula Rodríguez, M.D. ${ }^{a}$, Jesica Widmer, M.D. ${ }^{a}$ and Claudia Meregalli, M.D. ${ }^{a}$

\begin{abstract}
Multisystem inflammatory syndrome in children related to COVID-19 is defined as the presence of persistent fever, inflammation, and organ dysfunction, with evidence of past or recent severe acute respiratory syndrome coronavirus 2 infection, and excluding other microbial causes. It overlaps with other inflammatory diseases (Kawasaki disease and toxic shock syndrome) and shares some features with hypercytokinemia conditions (hemophagocytic lymphohistiocytosis and macrophage activation syndrome). It differs from these and severe acute COVID-19 in its clinical presentation and laboratory parameters. It has a potentially severe course and may occur with cardiovascular failure; mortality is low (2\%).

Here we provide an update on this syndrome and describe the presentation of two clinical cases with cardiovascular dysfunction who required vasoactive support and invasive ventilation. Serum lab tests showed inflammation parameters. Both patients were treated with intravenous immunoglobulin and systemic corticosteroids and had a favorable course.

Key words: Kawasaki disease, multisystem inflammatory syndrome in children temporally associated with SARS-CoV-2, MIS-C, multisystem inflammatory syndrome in children, COVID-19, SARS-CoV-2.
\end{abstract}

http: / / dx.doi.org/ 10.5546/ aap.2021.eng.e26

a. Department of Intensive Care of Hospital General de Niños "Dr. Pedro de Elizalde" (HGNPE), Autonomous City of Buenos Aires, Argentina.

E-mail address:

Pedro Taffarel, M.D.: pedrotaffarel@hotmail. com

Funding:

None.

Conflict of interest:

None.

Received: 8-11-2020

Accepted: 9-9-2020

\section{INTRODUCTION}

The incidence and severity of the pandemic caused by severe acute respiratory syndrome coronavirus 2 (SARS-CoV-2) are lower in the pediatric population compared to the adult population. ${ }^{1}$ The initial reports limited to the field of pediatrics state that mortality ranges from $0.1 \%$ to $4 \%$, with a severe clinical presentation similar to that of adult patients with coronavirus disease 2019 (COVID-19), which is characterized by acute respiratory distress syndrome (ARDS) and multiple organ failure, mostly in patients with comorbidities. ${ }^{2-9}$

In May 2020, cases of multiorgan involvement with circulatory shock and systemic inflammation were first reported in 8 children with COVID-19. ${ }^{10}$ The characteristics of this syndrome overlapped with Kawasaki disease (KD), toxic shock syndrome (TSS), hemophagocytic lymphohistiocytosis, and macrophage activation syndrome. The Royal College of Pediatrics and Child Health $(\mathrm{RCPCH})^{11}$ and the Centers for Disease Control and Prevention (CDC $)^{12}$ defined this disease as multisystem inflammatory syndrome in children related to COVID-19 (MIS-C) and published its diagnostic criteria (Table 1). In the United Kingdom, this condition is known as Paediatric Inflammatory Multisystem Syndrome Temporally Associated with SARSCoV-2 (PIMS-TS), whose case definition is less restrictive. ${ }^{13}$

The different published series indicate that mortality due to MIS-C is close to $2 \%$, with a high incidence of admissions to the pediatric intensive 
care unit (PICU) and a high rate of multiple organ support therapy. ${ }^{13-20}$ Guidelines developed by the American College of Rheumatology ${ }^{21}$ and a recent consensus have been published as guidance to the diagnostic approach of MIS-C.22 Both guidelines recommend organ support, stepwise progression of immunomodulatory therapy based on disease course and treatment response, and antiplatelet and / or anticoagulation therapy. ${ }^{21,22}$

Our objective is to describe the presentation, course, and treatment implemented in two patients with MIS-C and to perform a bibliographic review of this syndrome.

\section{CLINICAL CASE PRESENTATIONS}

Case 1: Previously healthy 4-year-old patient with a weight of $16 \mathrm{~kg}$ and a height of $103 \mathrm{~cm}$, a history of positive COVID-19 in the previous 4 weeks, few symptoms (fatigue and odynophagia), negative reverse transcription polymerase chain reaction (RT-PCR) for SARS-CoV-2, and positive immunoglobulin $\mathrm{G}(\mathrm{IgG})$ upon admission. The patient sought care due to persistent fever for 48 hours and cervical lymphadenopathy.

The patient was admitted to the Pediatric Ward and, 24 hours later, developed generalized rash and hemodynamic involvement, with a $27 \%$ fractional shortening observed in the echocardiogram. The patient was admitted to the PICU with cardiogenic shock and hypotension refractory to volume $(40 \mathrm{~mL} / \mathrm{kg})$. The patient required invasive mechanical ventilation (MV) due to impaired sensorium, inotropic support (epinephrine and norepinephrine at 0.1 and $0.25 \mathrm{mcg} / \mathrm{kg} /$ minute, respectively, as maximum dose), immunomodulatory therapy, and antiplatelet and anticoagulation therapy (acetylsalicylic acid and low molecular weight heparin [LMWH] at $1 \mathrm{mg} / \mathrm{kg} /$ day).

Immunosuppressive therapy was started upon admission to the PICU and systemic corticosteroids (dexamethasone at $0.15 \mathrm{mg} / \mathrm{kg} /$ day) and intravenous immunoglobulin (IVIG) at $2 \mathrm{~g} / \mathrm{kg} /$ dose were administered concomitantly. A second dose was required at 24 hours due to persistent fever and vasoactive drug requirement. The lab tests indicated systemic inflammation (Figure 1). The patient had a favorable course and was weaned from MV and cardiovascular support on the fourth day; in total, the patient stayed at the PICU for 7 days.

Case 2: Previously healthy 5-year-old patient with a weight of $20 \mathrm{~kg}$ and a height of $110 \mathrm{~cm}$, no history of past COVID-19 infection nor close contact with a positive COVID-19 patient, who developed fever, abdominal pain, vomiting, and generalized rash in the 7 days prior to admission. At the time of hospital admission, the patient was severely dehydrated and had conjunctival injection, cheilitis, bilateral cervical lymphadenopathy, and mild systolic ventricular dysfunction as per echocardiogram. The patient progressed to hypotension refractory to fluids, was admitted to the PICU, and required inotropic support (norepinephrine at $0.3 \mathrm{mcg} / \mathrm{kg} /$ minute, as maximum dose), MV, immunomodulatory therapy (IVIG at $2 \mathrm{~g} / \mathrm{kg}$ and dexamethasone at $0.15 \mathrm{mg} / \mathrm{kg} / \mathrm{day}$ ), and antiplatelet and anticoagulation therapy (acetylsalicylic acid and LMWH at $1 \mathrm{mg} / \mathrm{kg} /$ day).

The lab tests indicated a systemic inflammatory reaction (Figure 1). The patient's mother had a positive RT-PCR for SARS-CoV-2, while the child tested negative in the RT-PCR

TABLE 1. Case definition of multisystem inflammatory syndrome in children*

An individual aged $<21$ years presenting with fever, ${ }^{\text {a }}$ laboratory evidence of inflammation, ${ }^{\text {b }}$

and evidence of clinically severe disease requiring hospitalization, with multisystem (>2)

organ involvement (cardiac, renal, respiratory, hematologic, gastrointestinal, dermatologic or neurological)

and

no alternative plausible diagnoses

and

positive for current or recent SARS-CoV-2 infection by RT-PCR, serology, or antigen test; or COVID-19 exposure within the 4 weeks prior to the onset of symptoms.

\footnotetext{
${ }^{\text {a }}$ Fever $>38.0^{\circ} \mathrm{C}$ for $\geq 24$ hours, or report of subjective fever lasting $\geq 24$ hours.

${ }^{b}$ One or more of the following: an elevated C-reactive protein (CRP), erythrocyte sedimentation rate (ESR), fibrinogen,

procalcitonin, D-dimer, ferritin, lactic acid dehydrogenase (LDH), interleukin 6 (IL-6), elevated neutrophils, reduced

lymphocytes, and low albumin.

* Modified from CDC Multisystem Inflammatory Syndrome in Children (MIS-C) Associated with Coronavirus Disease 2019.12
} 
twice, but had a positive SARS-CoV-2 serology (immunoglobulin $\mathrm{M}[\mathrm{IgM}]$ and immunoglobulin $\mathrm{G}$ [IgG]). The patient had a favorable course; the vasoactive drip was discontinued after 72 hours and MV was stopped 48 hours after its initiation. The patient stayed at the PICU for 4 days.

None of these two patients showed pulmonary involvement in the chest X-ray, and their electrocardiograms and renal and liver function tests were normal. At the time of hospital discharge, a weekly follow-up appointment was scheduled with the Department of Rheumatology, with a progressive reduction in the systemic corticosteroid dose.

\section{RESULTS OF PUBLISHED CASE SERIES}

Since the onset of this new entity, several reports have been published. The main series described are compiled and summarized in Table $2 .{ }^{13-20}$ Whittaker et al. ${ }^{19}$ described three clinical course patterns in 58 patients with MIS-C: a group $(\mathrm{N}=23)$ made up of patients with persistent fever and high inflammatory markers, but without organ failure or mucocutaneous

\section{Case 1 Case 2}

\begin{tabular}{|c|c|c|}
\hline CRP (mg/L) & 245 & 114 \\
\hline $\begin{array}{l}\text { Erythrocyte sedimentation } \\
\text { rate }(\mathrm{mm} / \mathrm{h})\end{array}$ & 29 & - \\
\hline Procalcitonin (ng/mL) & 12.5 & 25.4 \\
\hline Fibrinogen $(\mathrm{mg} / \mathrm{dL})$ & 678 & 371 \\
\hline IL-6 (pg/mL) & 20 & - \\
\hline Platelets $\left(\times 10^{3} / \mathrm{mcl}\right)$ & 95 & 83 \\
\hline Lymphocytes (x 103/mcl) & 1.125 & 1.023 \\
\hline
\end{tabular}

\section{NT-proBNP (pg/mL)}

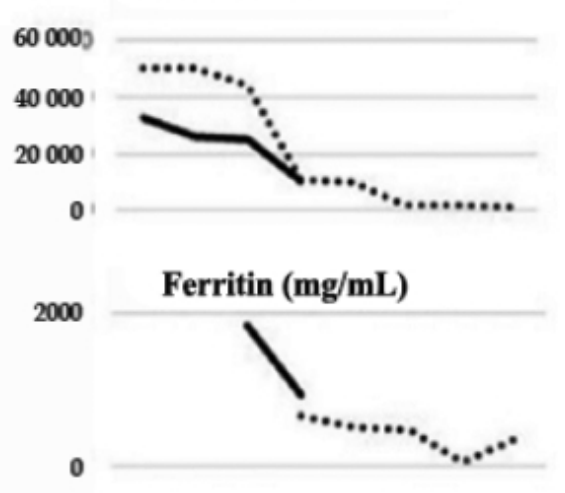

Troponin $(\mathrm{ng} / \mathrm{mL})$

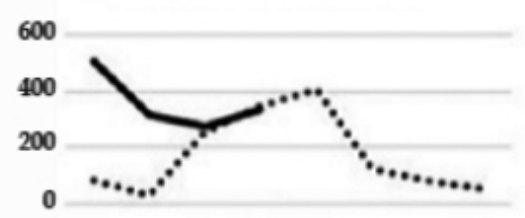

D-dimer (meg/mL)

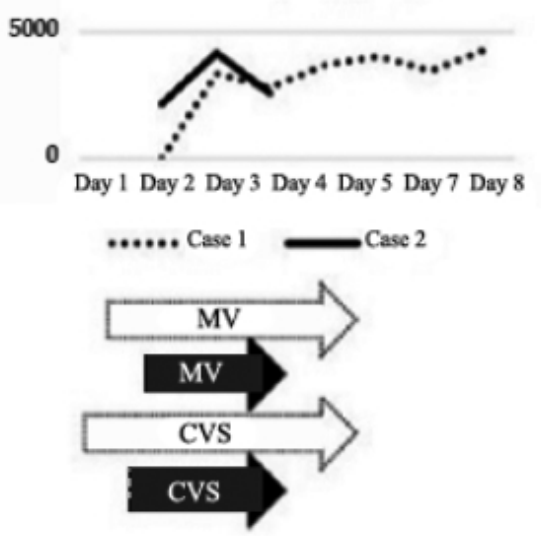

CRP: C-reactive protein; DD: D-dimer; NT-proBNP: N-terminal pro-B-type natriuretic peptide; IL-6: interleukin 6; MV: mechanical ventilation; CVS: cardiovascular support. 
characteristics suggestive of KD or TSS; a second group $(\mathrm{N}=29)$ of patients who developed shock with evidence of left ventricular dysfunction and high myocardial markers (troponin and $\mathrm{N}$-terminal pro-B-type natriuretic peptide [NTproBNP]), and a third group $(\mathrm{N}=7)$ of patients who met the diagnostic criteria of the American Heart Association (AHA) for KD. One of these 7 patients progressed to shock, so they were also registered in the former group.

Patients with MIS-C described in this series were compared to a historical cohort of patients with other inflammatory diseases (1132 patients with KD, 45 with KD shock syndrome, and 37 with TSS). When the patients described by Whittaker ${ }^{19}$ were compared to those with KD and KD shock in the historical cohort, they were older, had higher CRP values, leukocytosis, neutrophilia, lymphopenia, anemia, and thrombocytopenia, and higher fibrinogen and troponin levels. When the patients with MIS-C $(\mathrm{N}=58)$ were compared to the historical cohort of patients with TSS $(\mathrm{N}=37)$, they were also older (9 years old [5.7-14] in the MIS-C group and 7.4 years old [2.4-15.4] in the TSS group) and had lower hemoglobin levels.

The recent consensus on PIMS-TS divides the clinical presentation into two phenotypes: Kawasaki disease-like (complete or incomplete) and non-specific, i.e. children presenting with shock and/or fever and gastrointestinal, respiratory or neurological symptoms that do not meet KD criteria. ${ }^{22}$

Sixty-eight percent of patients reported in the different series ${ }^{13-20}$ analyzed in this article $(\mathrm{N}=1092)$ did not have a prior disease. Overweight and obesity were the main concomitant conditions. Fever is the cardinal symptom of this syndrome, present in $99.8 \%$ of cases, followed, in order of frequency, by the following signs and symptoms: gastrointestinal manifestations in $94.2 \%$, mucocutaneous manifestations in $64.5 \%$, and conjunctivitis in $63 \%$.

The definition of PIMS-TS is less strict in terms of recent or past SARS-CoV-2 infection, so some patients analyzed in the series by Davies ${ }^{13}$ (33 out of 78) and Whittaker ${ }^{19}$ (13 out of 58) did not meet the MIS-C criteria. The etiological association was documented by positive PCR and/or serology for SARS-CoV-2 in $47 \%$ and $62 \%$, respectively, in the 1092 patients described in the 8 series..$^{13-20}$

Inflammatory markers were high in all analyzed series. The average values of the different reactants were CRP: $202 \mathrm{mg} / \mathrm{L}$; D-dimer (DD): $2986 \mathrm{mg} / \mathrm{mL}$; procalcitonin: $15 \mathrm{mg} / \mathrm{mL}$; ferritin: $550 \mathrm{mg} / \mathrm{mL}$; and fibrinogen: $598 \mathrm{mg} / \mathrm{dL}$. The incidence of cardiovascular involvement was high in the different reports; clinical characteristics of shock were observed in $43.3 \%$ of all patients; myocardial involvement based on high troponin levels was documented in $31 \%, 50 \%$, and $71 \%$ of the patients in the series by Godfred-Cato, ${ }^{20}$ Feldstein, ${ }^{16}$ and Dufort, ${ }^{17}$ respectively. The average values of these myocardial inflammatory markers were $119 \mathrm{mg} / \mathrm{L}$ for troponin and $2656 \mathrm{pg} / \mathrm{mL}$ for NTproBNP. Left ventricular dysfunction as observed in the echocardiogram was present in $42 \%$ of patients. Coronary artery abnormalities were observed in $18.5 \%$ of all cases. ${ }^{13-20}$

The analysis of the different series suggests that MIS-C has a severe presentation with a high rate of organ support requirement. Out of the 1092 patients described in the main 8 series, ${ }^{13-20} 49 \%$ required vasoactive support; $19.2 \%$, invasive $\mathrm{MV}$; and $5.5 \%$, extracorporeal membrane oxygenation (ECMO) (in the 7 series that specified its use, $N=522$ ). In relation to immunomodulatory therapy, $58 \%$ of patients received systemic corticosteroids; $74 \%$, IVIG; and $22 \%$, biological agents, anakinra, tocilizumab and/or infliximab (the first two were most commonly used). The overall mortality rate in the 1092 analyzed cases was $1.7 \%$.

In the largest series $(\mathrm{N}=570)^{20}$ described in children with MIS-C, the authors performed a latent class analysis (LCA), a statistical modeling technique that allowed to divide cases into groups with underlying similarities. The indicator outcome measures used in the LCA were presence or absence of positive RT-PCR or serology for SARS-CoV-2, shock, pneumonia, and organ or system involvement (cardiovascular, dermatologic, gastrointestinal, hematologic, neurological, renal or respiratory). This way, individuals were divided into three different classes:

Class $1(\mathrm{~N}=203,35.61 \%)$ included patients with the highest number of affected organs and systems, a higher prevalence of abdominal pain, shock, myocarditis, lymphopenia, markedly high CRP, ferritin, troponin, B-type natriuretic peptide (BNP) or pro-BNP, and positive SARS-CoV-2 serology (98\%) with or without positive RT-PCR for SARS-CoV-2. Class $2(\mathrm{~N}=169,29.65 \%)$ included patients with respiratory system involvement $(76.3 \%)$, a significantly higher rate of positive RT- 


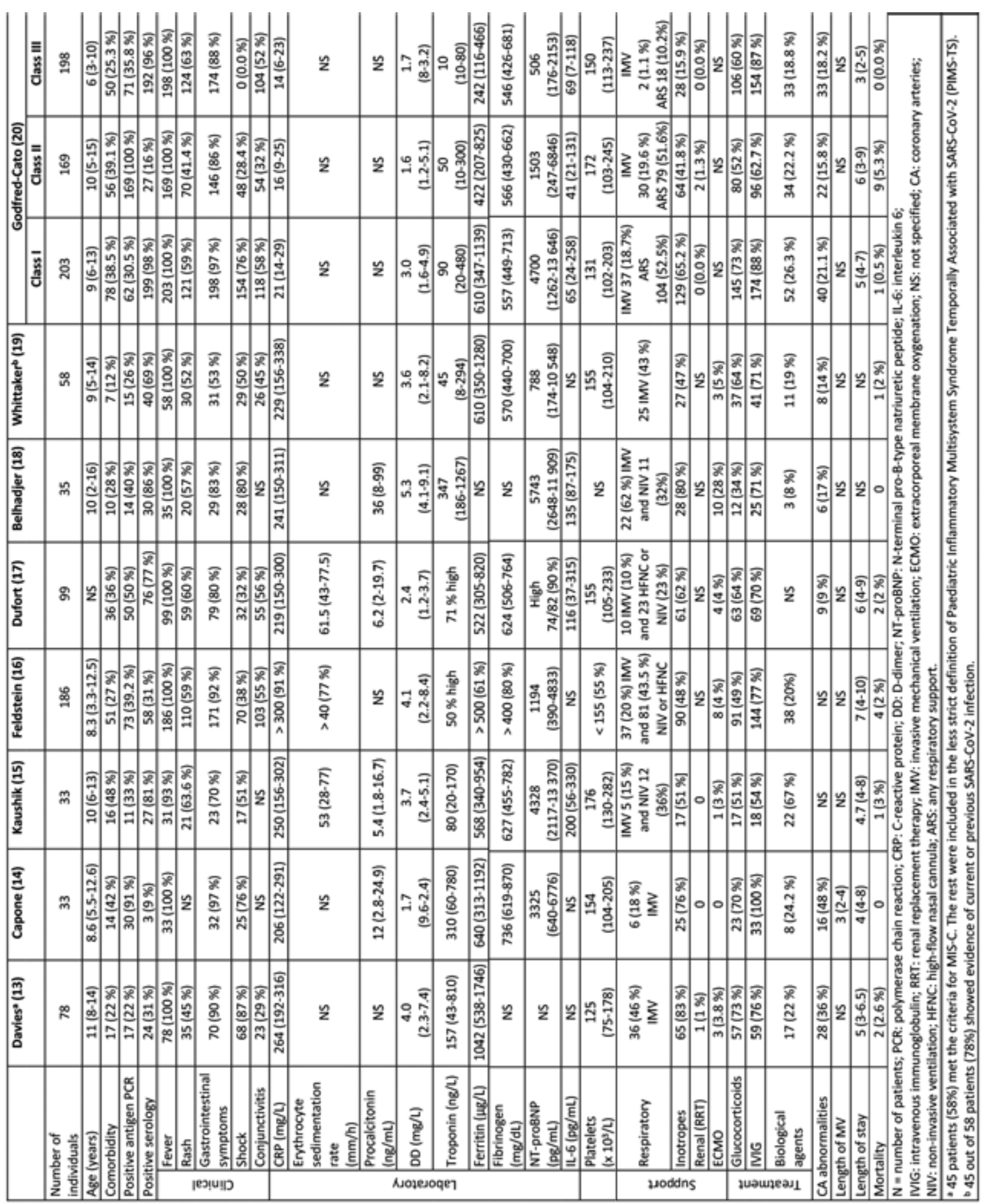


PCR for SARS-CoV-2 (without positive serology) $(84.0 \%)$ than patients in class $1(0.5 \%)$ or class 3 $(2.0 \%)(p<0.01)$, and a higher fatality rate. Class 3 $(\mathrm{N}=198,34.74 \%)$ included younger children with a higher prevalence of rash and mucocutaneous lesions who most commonly met the criteria for complete KD (6.6\%).

\section{DISCUSSION}

The differences in the definition of this syndrome as proposed by the different entities are small. The one used by the National Ministry of Health of Argentina ${ }^{23}$ is that proposed by the World Health Organization (WHO) ${ }^{24}$ and differs from that used by the CDC, which is mentioned in this article (Table 1), in relation to the younger age of patients ( 0 to 18 years) and the longer duration of fever (more than 3 days). In addition, a documented recent or past SARSCoV-2 infection (RT-PCR and/or serology) is a requirement of the National Ministry of Health of our country, whereas according to both the WHO and the CDC, exposure to COVID-19 or potential contact with a COVID-19 patient is enough.

The publication of the early reports on MIS-C raised the question about whether this was a new disease, a variant of acute COVID-19 presentation or an exacerbation of the frequency of known inflammatory syndromes resulting from the pandemic. Overlapping KD symptoms may lead to diagnostic uncertainty. Therefore, different series included KD in the reports of patients with MIS-C because they met KD diagnostic criteria. However, unlike the usual presentation of KD, the one associated with SARS-CoV-2 occurs in older patients with worse cardiac failure and resistance to immunoglobulins, observed based on the Kobayashi score. ${ }^{25,26}$ This allows to predict a lack of response to IVIG in KD based on 7 variables (age, neutrophil percentage, platelet count, serum aspartate aminotransferase, blood sodium, CRP, and length of fever in days), considering patients with a score of 4 or more to be high risk. ${ }^{27}$

In relation to the clinical characteristics of MIS-C, it particularly affects previously healthy patients, unlike acute COVID-19 infection, whose most severe presentation is strictly related to the presence of comorbidities. In addition to aid in diagnosis, a hematological and biochemical profile and heart assessment allow to evaluate disease progression and guide its management.

Regarding the classes proposed for MIS-C presentation by Godfred-Cato et al. ${ }^{20}$ children in class 1 had a clinical course compatible with previous reports of MIS-C that did not overlap with acute COVID-19 or KD. Patients in class 2 had a greater involvement of the respiratory system, indicating that the disease could have been mainly acute COVID-19 or a combination of COVID-19 and MIS-C, which resulted in a higher fatality rate $(5.3 \%)$ across the 3 classes $(p<0.01)$. Class 3 showed a greater relation with typical KD and was observed in younger patients with a greater mucocutaneous involvement and a higher frequency of coronary artery aneurysm. Both cases described in this article correspond to class 1, as proposed by Godfred-Cato. ${ }^{20}$

Certain clinical and laboratory characteristics may help to differentiate MIS-C from KD secondary to SARS-CoV-2. Abdominal pain, lymphopenia, high NT-proBNP levels, and myocardial dysfunction as per echocardiogram are common in MIS-C. ${ }^{28}$ In addition, the inflammatory response in MIS-C is similar to that of $\mathrm{KD}$, but it differs from the latter in the subsets of T cells, IL-17A, and biomarkers associated with artery damage. The autoantibody profile suggests that endoglin, an endothelial glycoprotein, is one of several targets of autoantibodies in MIS-C. ${ }^{29}$

The two cases described in this article are related to the clinical presentation and progression observed in the different analyzed series, with a significant short-term morbidity, in terms of critical care requirements, but with a low mortality rate and a favorable course.

Treatment will depend on affected organs, whereas the specific treatment of this syndrome is based on immunomodulators. The onset time and progression are the result of disease course and therapeutic response. Harwood et al. segmented the treatment based on the presenting phenotype (KD or non-specific). ${ }^{22}$ In both sub-types, IVIG is the first-line treatment $(2 \mathrm{~g} / \mathrm{kg})$, and a second dose may be needed. Low-dose corticosteroids are proposed as an optional treatment in the case of both phenotypes.

Systemic corticosteroids (methylprednisolone) are the second-line treatment in the case of lack of response to IVIG ( 24 hours), and persistent fever is its main marker. Among patients with KD phenotype who are younger than 12 months or have coronary artery involvement, the first and second lines are administered in combination. Our patients received corticosteroid therapy in the form of dexamethasone equivalent to $1 \mathrm{mg} /$ $\mathrm{kg} /$ day of prednisolone.

Biological agents constitute the third line of treatment. Infliximab is indicated for the KD 
phenotype, whereas there is no consensus among tocilizumab, anakinra, and infliximab for the nonspecific presentation. ${ }^{22}$ The recommendations mentioned in the bibliography are summarized in Figure 2.

\section{FINAL COMMENT}

In both case reports, MIS-C presented as a florid inflammatory condition in relation to a past SARS-CoV-2 infection. Both patients showed cardiovascular involvement, so they required hemodynamic and respiratory support. The response to immunomodulatory therapy was favorable in both cases.

Since pediatric patients often present with mild or no COVID-19 symptoms, it has not been possible to establish the incidence of MIS-C in children with SARS-CoV-2 infection. Therefore, it is critical to implement a strict surveillance for case screening, which requires lab tests, ancillary studies, including an echocardiogram, and multidisciplinary follow-up (by the departments of infectology, cardiology, rheumatology, intensive care), in order to guide the diagnosis and management of suspected cases.

FIGURE 2. Diagnostic and therapeutic algorithm for patients with multisystem inflammatory syndrome in children

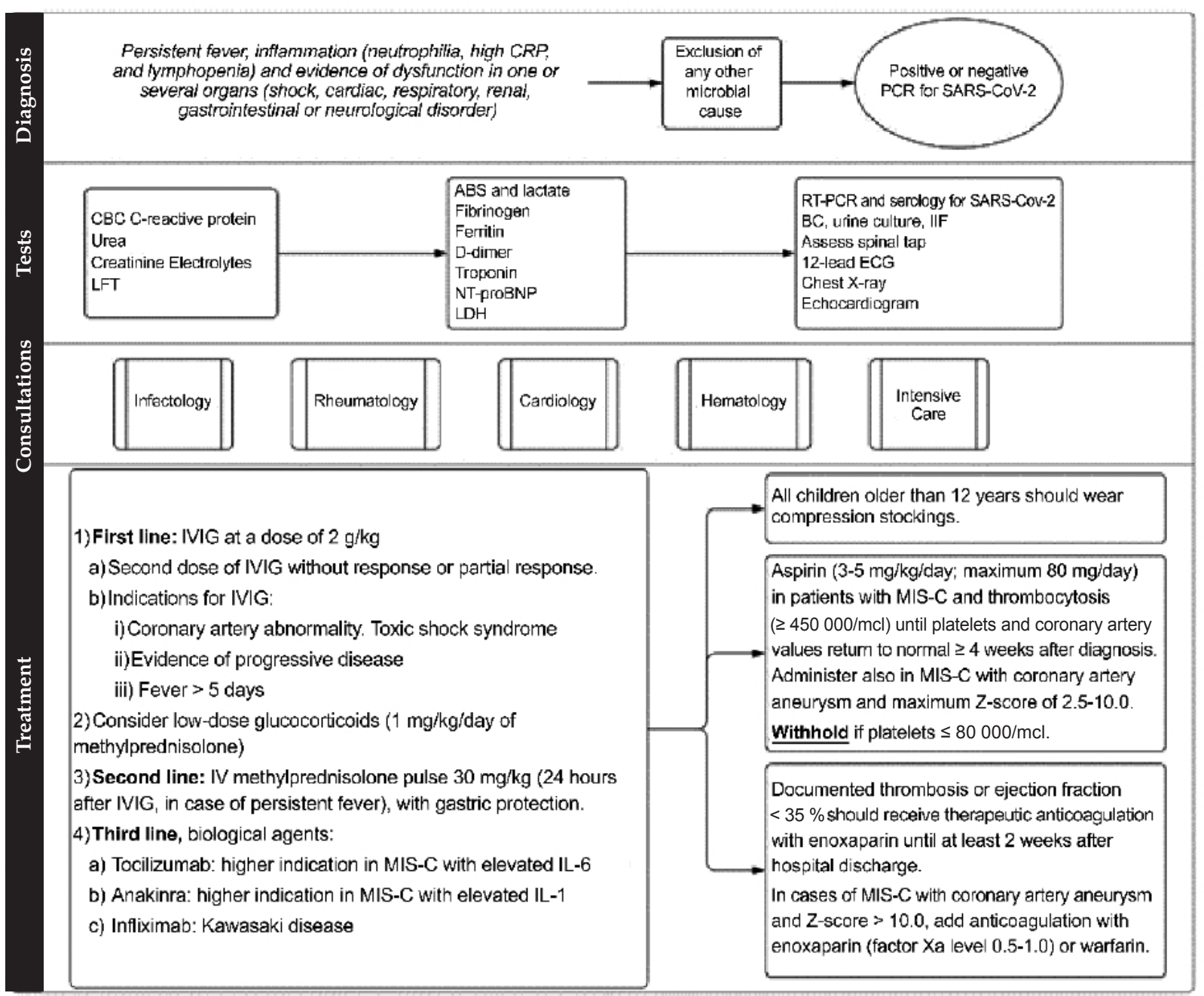

RT-PCR: reverse transcription polymerase chain reaction; CBC: complete blood count; LFT: liver function tests; ABS: acid-base status; NT-proBNP: N-terminal pro-B-type natriuretic peptide; LDH: lactate dehydrogenase; BC: blood cultures; IIF: indirect immunofluorescence of nasopharyngeal secretions; ECG: electrocardiogram;

IVIG: intravenous immunoglobulin; IL: interleukin.

Obtained and modified from the American College of Rheumatology ${ }^{21}$ and Harwood R..$^{22}$ 


\section{REFERENCES}

1. Ong JSM, Tosoni A, Kim Y, Kissoon N, et al. Coronavirus Disease 2019 in Critically Ill Children: A Narrative Review of the Literature. Pediatr Crit Care Med. 2020; 21(7):662-6.

2. Parri N, Lenge M, Buonsenso D. Children with Covid-19 in Pediatric Emergency Departments in Italy. N EnglJ Med. May 2020; 383(2):187-90.

3. Dong $Y, M o X, H u Y, Q i X$, etal.EpidemiologicalCharacteristics of 2143 Pediatric Patients With 2019 Coronavirus Disease in China. Pediatrics. 2020. [Accessed on: September 9 ${ }^{\text {th }}, 2020$ ]. Available at: https://pediatrics.aappublications.org/ content/ pediatrics / early / 2020 / 03 / 16/peds.2020-0702. full.pdf.

4. Lu X, Zhang L, Du H, Zhang J, et al. SARS-CoV-2 Infection in Children. N Engl J Med. 2020; 382(17):1663-5.

5. Castagnoli R, Votto M, Licari A, Brambilla I, et al. Severe Acute Respiratory Syndrome Coronavirus 2(SARS-CoV-2) Infection in Children and Adolescents: a systematic review. JAMA Pediatr. 2020; 174(9):882-9.

6. CDC COVID-19 Response Team. Coronavirus Disease 2019 in Children - United States, February 12-April 2, 2020. MMWR Morb Mortal Wkly Rep. 2020; 69(14):422-6.

7. Shekerdemian LS, Mahmood NR, Wolfe KK, Riggs B, et al. Characteristics and Outcomes of Children With Coronavirus Disease 2019 (COVID-19) Infection Admitted to US and Canadian Pediatric Intensive Care Units. JAMA Pediatr. 2020; 174(9):868-73.

8. González-Dambrauskas S, Vásquez-Hoyos P,Camporesi A, Díaz-Rubio F, et al. Pediatric Critical Care and COVID19. Pediatrics. 2020; 146(3):e20201766.

9. Götzinger F, Santiago-García B, Noguera-Julián A, Lanaspa M, et al. COVID-19 in children and adolescents in Europe: a multinational, multicentre cohort study. Lancet Child Adolesc Health. 2020; 4(9):653-61.

10. Riphagen S, Gomez X, Gonzalez-Martinez C, Wilkinson $\mathrm{N}$, et al. Hyperinflammatory shock in children during COVID-19 pandemic. Lancet. 2020; 395(10237):1607-8.

11. Royal College of Paediatrics and Child Health. Guidance: Paediatricmultisystem inflammatory syndrome temporally associated with COVID-19. [Accessed on: July 18 ${ }^{\text {th }}, 2020$ ]. Available at: https://www.rcpch.ac.uk/sites/default/ files / 2020-05 / COVID-19-Paediatric-multisystem-\%20 inflammatory \%20syndrome-20200501.pdf.

12. Centers for Disease Control and Prevention. Multisystem Inflammatory Syndrome in Children (MIS-C) Associated with Coronavirus Disease 2019. 2020. [Accessed on: July 17 $\left.{ }^{\text {th }}, 2020\right]$. Available at: https://emergency.cdc.gov/ han/2020/han00432.asp.

13. Davies P, Evans C, Kanthimathinathan HK, Lillie J, et al. Intensive care admissions of children with paediatric inflammatory multisystem syndrome temporally associated with SARS-CoV-2 (PIMS-TS) in the UK: a multicentre observational study. Lancet Child Adolesc Health. 2020; 4(9):669-77.

14. Capone CA, Subramony A, Sweberg T, Schneider J, et al. Characteristics, Cardiac involvement, and Outcomes of Multisystem Inflammatory Disease of Childhood (MIS-C) Associated with SARS-CoV-2 Infection. J Pediatr. 2020; 224:141-5.

15. Kaushik S, Aydin SI, Derespina KR, Bansal PB, et al. Multisystem Inflammatory Syndrome in Children Associated with Severe Acute Respiratory Syndrome Coronavirus 2 Infection: A Multi-institutional Study from New York City. J Pediatr. 2020; 224:24-9.
16. Feldstein LR, Rose EB, Horwitz SM, Collins JP, et al. Multisystem Inflammatory Syndrome in U.S. Children and Adolescents. N Engl J Med. 2020; 383(4):334-46.

17. Dufort EM, Koumans EH, Chow EJ, Rosenthal EM, et al. Multisystem Inflammatory Syndrome in Children in New York State. N Engl J Med. 2020; 383(4):347-58.

18. Belhadjer Z, Méot M, Bajolle F, Khraiche D, et al. Acute heart failure in multisystem inflammatory syndrome in children (MIS-C) in the context of global SARS-CoV-2 pandemic. Circulation. 2020; 142(5)429-36.

19. Whittaker E, Bamford A, Kenny J, Kaforou M, et al. Clinical Characteristics of 58Children with a Pediatric Inflammatory Multisystem Syndrome Temporally Associated withSARSCoV-2. JAMA. 2020; 324(3):259-69.

20. Godfred-Cato S, Bryant B, Leung J, Oster M, et al. COVID19-Associated Multisystem Inflammatory Syndrome in Children - United States, March-July 2020. MMWR Morb Mortal Wkly Rep. 2020; 69(32):1074-80.

21. Henderson LA, Canna SW, Friedman KG, Gorelik M, et al. American College of Rheumatology Clinical Guidance for Pediatric Patients with Multisystem Inflammatory Syndrome in Children (MIS-C) Associated with SARSCoV-2 and Hyperinflammation in COVID-19. Version 1. Arthritis Rheumatol. [Accessed on: August $8^{\text {th }}, 2020$ ]. Available at:https://www.rheumatology.org/Portals/0/ Files/ACR-COVID-19-Clinical-Guidance-Summary-MISC-Hyperinflammation.pdf.

22. Harwood R, Allin B, Jones CE, Whittaker E, et al. A national consensus management pathway for PaediatricInflammatory Multisystem Syndrome - Temporally associated with SARSCoV-2 (PIMS-TS): The results of a national Delphi process. [Accessed on: August $\left.8^{\text {th }}, 2020\right]$. Available at: https: / / www. medrxiv.org/content/10.1101/2020.07.17.20156075v1.full. pdf.

23. Argentina. Ministerio de Salud. Vigilancia, diagnóstico y manejo institucional de casos en pediatría. [Accessed on: September $\left.7^{\text {th }}, 2020\right]$. Available at: https: / / www.argentina. gob.ar/salud/ coronavirus-COVID-19/ casos-pediatria.

24. World Health Organization. Multisystem inflammatory syndrome in children and adolescents with COVID-19: scientific brief, 15 May 2020. [Accessed on: September $\left.7^{\text {th }}, 2020\right]$. Available at: https://apps.who.int/iris/ handle/10665/332095.

25. Verdoni L, Mazza A, Gervasoni A, Martelli L, et al. An outbreak of severe Kawasaki-like disease at the Italian epicentre of the SARS-CoV-2 epidemic: an observational cohort study. Lancet. 2020; 395(10239):1771-8.

26. Ouldali N, Pouletty M, Mariani P, Beyler C, et al. Emergence of Kawasaki disease related to SARS-CoV-2 infection in an epicentre of the French COVID-19 epidemic: a time-series analysis. Lancet Child Adolesc Health. 2020; 4(9):662-8.

27. Kobayashi $\mathrm{T}$, Inoue $\mathrm{Y}$, Takeuchi $\mathrm{K}$, Okada $\mathrm{Y}$, et al. Prediction of intravenousimmunoglobulinunresponsivenessin patients with Kawasaki disease. Circulation. 2006; 113(22):2606-12.

28. Rowley AH. Multisystem Inflammatory Syndrome in Children and Kawasaki Disease: Two Different Illnesses with Overlapping Clinical Features. J Pediatr. 2020; 224:12932.

29. Rosat Consiglio C, Cotugno N, Sardh F, Pou C, et al. The Immunology of Multisystem Inflammatory Syndrome in Children with COVID-19. [Accessed on: August $8^{\text {th }}, 2020$ ]. Available at: https: / / www.medrxiv.org/content/10.1101 / 2020.07.08.20148353v2. 